\title{
Model Checking as A Reachability Problem
}

\author{
Moshe Y. Vardi ${ }^{\star}$ \\ Rice University, Department of Computer Science, Houston, TX 77251-1892, U.S.A. \\ vardiacs.rice.edu \\ http: //www.cs.rice.edu/ vardi
}

\begin{abstract}
Model checking is a essentially a graph-searching problem. In automata-theoretic model checking we compose the design under verification with a Büchi automaton that accepts traces violating the specification. We then use graph algorithms to search the product graph for a counterexample trace. The basic theory of this approach was worked out in the 1980s, and the basic algorithms were developed during the 1990s. Both explicit and symbolic implementations, such as SPIN and and SMV, are widely used. It turns out, however, that there are still many gaps in our understanding of the algorithmic issues involved in automata-theoretic model checking. This talk covers the fundamentals of automata-theoretic model checking, reviews recent progress, and outlines areas that require further research.
\end{abstract}

\section{References}

1. Vardi, M.Y.: An automata-theoretic approach to linear temporal logic. In: Moller, F., Birtwistle, G. (eds.) Logics for Concurrency. LNCS, vol. 1043, pp. 238-266. Springer, Heidelberg (1996)

2. Vardi, M.Y.: Automata-theoretic model checking revisited. In: Cook, B., Podelski, A. (eds.) VMCAI 2007. LNCS, vol. 4349, pp. 137-150. Springer, Heidelberg (2007)

\footnotetext{
* Supported in part by NSF grants CCR-0124077, CCR-0311326, CCF-0613889, ANI-0216467, and CCF-0728882, by BSF grant 9800096, and by gift from the Intel Corporation.
} 